\title{
e-Phaïstos
}

e-Phaïstos

Revue d'histoire des techniques / Journal of the history

of technology

V-1 2016 | 2018

Ville et technique

\section{Application du concept de filière technique aux instruments de musique : les instruments à vent en corne médiévaux et modernes}

Conceptual application of sector techniques applicated to music instruments :

Medevial and modern wind musical instruments

\section{Marion Weckerle}

\section{OpenEdition}

Journals

Édition électronique

URL : http://journals.openedition.org/ephaistos/1251

DOI : 10.4000/ephaistos.1251

ISSN : 2552-0741

Éditeur

IHMC - Institut d'histoire moderne et contemporaine (UMR 8066)

Référence électronique

Marion Weckerle, «Application du concept de filière technique aux instruments de musique : les instruments à vent en corne médiévaux et modernes », e-Phaïstos [En ligne], V-1 2016 | 2018, mis en ligne le 21 janvier 2018, consulté le 19 avril 2019. URL : http://journals.openedition.org/ ephaistos/1251; DOI : 10.4000/ephaistos.1251

Ce document a été généré automatiquement le 19 avril 2019

Tous droits réservés 


\section{Application du concept de filière technique aux instruments de musique : les instruments à vent en corne médiévaux et modernes} Conceptual application of sector techniques applicated to music instruments :
Medevial and modern wind musical instruments

Marion Weckerle

\section{Introduction}

1 Contrairement à ce qu'une pensée intuitive pourrait faire croire, pour confectionner un instrument de musique ou en jouer, la nature du matériau, même si elle influe sur le son, n'est pas un facteur conditionnant en premier lieu les techniques appliquées. Ainsi, une variété d'instruments relevant de différentes filières techniques, et qui s'insèrent dans des contextes musicaux très différents, peuvent prendre corps, à partir d'un même matériau. La plupart des instruments à vent anciens étaient réalisés en bois ou en matières dures animales, c'est-à-dire principalement l'os, l'ivoire et la corne ${ }^{1}$; ceux en corne, très peu étudiés, constituent un objet technique tout à fait pertinent pour étudier la question des filières techniques, tout en apportant des éléments nouveaux à la pratique de la musique ancienne historiquement informée. Le concept de filière technique, tel que développé au sein de l'équipe d'histoire des techniques de l'Université Paris 1 par AnneFrançoise Garçon, se définit comme une logique, un fonctionnement technique partagé par différents objets.

2 Nous avons relevé cinq types d'instruments propres à se prêter à cette étude, dans l'aire géographique de l'Europe et du Proche-Orient : la pifana ou pivana (cf illustration 1), le hornpipe (cf illustration 2), le gemshorn (ou moins couramment cor de chamois en français, cf illustration 3), le cor d'appel et le shofar (ou chofar, שופ en hébreu). Les trois 
premiers sont à rapprocher des flûtes, tandis que les deux derniers sont des instruments à embouchure à rapprocher des cuivres ${ }^{2}$. Tous partagent une existence temporelle longue puisqu'ils étaient très probablement tous joués avant le Moyen Âge, tout en connaissant désormais un usage contemporain confidentiel voire exceptionnel mais mêlé d'un regain d'intérêt ces dernières années.

3 Le hornpipe est en réalité un terme correspondant à une catégorie d'instruments de différentes régions (pibole en français, pibgorn, pibcorn ou piccorn au pays de Galles ${ }^{3}$, stock-and-horn en Écosse ${ }^{4}$, alboka au pays basque), définie par un tuyau en matière végétale ou en os percé de trous, à anche simple, avec une capsule protégeant l'anche et un pavillon tous deux faits de corne ${ }^{5}$. Le cor d'appel est ici employé pour désigner l'olifant (ou oliphant), lequel terme sous-entend généralement un instrument en ivoire ou en os, mais pouvant prêter à confusion. Le simple terme de cor n'étant lui pas assez explicite (comme pour le terme «horn » en anglais ou en allemand), utiliser « cor d'appel » à la place lève ici l'ambiguïté.

En premier lieu, nous allons passer en revue l'historiographie et les sources disponibles relatives à ces instruments. En second lieu, nous expliciterons les problématiques et la méthodologie spécifiques qui se posent alors. Enfin, nous procéderons à l'analyse des résultats obtenus.

\section{Historiographie}

5 L'historiographie sur le gemshorn se résume à quelques articles se citant les uns les autres et peinant à apporter des informations nouvelles. La plus ancienne étude est un article de 1918 de l'Allemand Curt Sachs ${ }^{6}$, et rien n'est fait ensuite jusqu'au début des années 1970, où les auteurs sont confrontés à des difficultés d'identification et aux limites d'éléments bibliographiques trop anciens ${ }^{7}$, en confondant des matériaux voire des familles d'instruments (tournebout et gemshorn par exemple ${ }^{8}$ ). L'article de 1981 d'Andrew Parkinson réexamine le peu d'iconographie disponible mais sans parvenir à une réelle conclusion; il propose cependant sa propre méthode de construction d'un gemshorn à 4 trous et une tablature de doigtés9 .

La bibliographie du hornpipe est tout aussi discrète, majoritairement composée d'études d'anthropologie anciennes (avant 1970) passées inaperçues, à l'exception d'un article récent bien documenté ${ }^{10}$. Il s'agit de discuter des origines des hornpipes, en croisant éléments culturels issus de sources littéraires, et informations organologiques issues de l'archéologie. Henry Balfour propose un arbre généalogique englobant les différentes variantes de hornpipes et bagpipes, pour tenter d'expliquer cette lignée technique ${ }^{11}$. Le travail de Denis Le Vraux sur les piboles est intéressant à remarquer de par sa démarche relevant tout à fait de celles pratiquées en histoire des techniques lorsqu'elles touchent l'organologie ${ }^{12}$, et faisant appel à l'expérimentation. L'auteur propose une reconstitution et un essai d'un artefact archéologique de Mayenne initialement identifié comme étant une flûte médiévale et s'avérant d'après son analyse être une pibole ${ }^{13}$.

7 L'historiographie sur la pifana est inexistante ; elle est même absente des dictionnaires d'instruments les plus complets comme le Guide des instruments anciens ${ }^{14}$. On ne trouve que quelques mentions sur Internet en tant qu'instrument traditionnel corse, sans effort de documentation. Le cor d'appel est étudié en tant qu'ancêtre de la famille des aérophones à embouchure. Les principales sources sont littéraires (Chanson de Roland, Marin 
Mersenne) et iconographiques ${ }^{15}$, mais la documentation archéologique manque et il n'a pas été étudié en tant qu'instrument à part entière. Plusieurs ouvrages se tournent vers l'ethnologie africaniste pour pallier au manque d'informations et se documentent à la place sur les «trompes » en cornes d'antilopes ${ }^{16}$.

Le shofar tient une place à part dans l'historiographie musicale puisqu'il est systématiquement étudié dans son contexte cultuel. Son historiographie s'appuie largement sur l'Ancien Testament, et analyse en profondeur le symbolisme entourant l'instrument. Il est par contre absent d'ouvrages plus musicologiques ainsi que de la plupart des dictionnaires. Les quelques auteurs sont eux-mêmes sonneurs de shofar ${ }^{17}$, par exemple Jeremy Montagu, qui présente des indications techniques et une typologie via sa propre collections de shofars ${ }^{18}$.

\section{Sources disponibles}

Les traités de réduction en art du XVI ${ }^{e}$ siècle s'intéressent à la musique avant tout sous son aspect théorique, et beaucoup d'instruments populaires, rares ou désuets ne sont pas pris en compte. Ainsi, le gemshorn apparaît dans trois traités allemands de la première moitié du XVI ${ }^{\mathrm{e}}$ siècle ${ }^{19}$ mais ni son jeu ni sa fabrication ne sont détaillés, et il n'existe pas non plus de partition.

10 Les textes littéraires relevant de la mythologie, renseignent sur les valeurs culturelles. Dans la Bible, les traductions fantaisistes obligent à vérifier toutes les mentions du shofar dans le texte hébreu. La Chanson de Roland mentionne un cor ou olifant décoré associé au combat désespéré du héros ${ }^{20}$, comme dans Siegfried de Richard Wagner ${ }^{21}$ ou la Communauté de l'Anneau de J.R.R Tolkien ${ }^{22}$. Dans la littérature britannique, on rencontre probablement un gemshorn dans The Complaynt of Scotland (« ane pipe maid of ane gait horne »), ainsi qu'un hornpipe (" ane corne pipe »), apparaissant également dans The Sad Shepherd et le Romaunt of the Rose ${ }^{23}$.

11 Enfin, la principale source est matérielle: les instruments eux-mêmes conservés en musées. Le site internet Musical Instrument Museums Online recense en particulier les collections de trente musées européens ${ }^{24}$, mais ces instruments sont rares, la corne se conservant très mal.

L'iconographie représente généralement l'une des principales sources en organologie, mais nous l'écartons ici pour des raisons méthodologiques (cf. infra). Elle est très restreinte pour le gemshorn et le hornpipe, mais plus riche pour le cor d'appel et le shofar, seule celle de ce dernier instrument étant régulièrement mise à profit dans l'historiographie.

\section{Problématiques et méthodologie}

13 L'organologie se documentant largement à partir des instruments eux-mêmes, de l'iconographie et des partitions, types de sources rares ou absentes ici, une étude comme celle-ci est peu susceptible d'être menée dans ce champs disciplinaire. Il s'agit ici de comprendre les différents instruments choisis en termes de filières techniques, en relation avec le matériau "corne». Cela implique de décrire une partie du complexe technique ${ }^{25}$ de ces instruments ainsi que la place sociale de leur jeu. Deuxièmement, nous voulons tenter d'apporter des éléments pour des reconstitutions historiquement 
informées d'instruments ainsi qu'encourager la curiosité des ethnomusicologues. Enfin, nous souhaitons proposer un test méthodologique. Afin de ne pas surcharger l'article, nous ne proposons pas d'analyse iconographique, et les sources matérielles sont dans ce cas les plus évidentes et nécessaires pour une étude de ce type. Toutefois la documentation archéologique est très limitée, nous ne disposons pas de collection personnelle, et il est difficile d'accéder physiquement aux instruments conservés en musées. Nous nous proposons donc de travailler à partir des bases de données de musées en ligne, censées répondre à cette problématique, et d'en évaluer dans le même temps la pertinence.

\section{Analyse}

\section{Des animaux au matériau}

14 La corne utilisée peut provenir de plusieurs animaux différents (cf. tableau 1). Dans certains cas (gemshorn, pifana et shofar), l'instrument doit impérativement être en corne : le matériau participe à la définition de l'instrument. Ce n'est pas le cas pour le cor d'appel par exemple, qui existe aussi en ivoire, terre cuite ou grès.

Tableau 1 : Origine de la corne utilisée dans chaque type d'instrument

\begin{tabular}{|l|l|}
\hline Instrument & Animal \\
\hline Gemshorn & $\begin{array}{l}\text { Chamois } \\
\text { Vache domestique } \\
\text { Chèvre domestique } \\
\text { Bouquetin des Alpes } \\
\text { Bouquetin ibérique }\end{array}$ \\
\hline Pifana & Chèvre \\
\hline Hornpipe & $\begin{array}{l}\text { Vache domestique } \\
\text { Mouton } \\
\text { Cerf }\end{array}$ \\
\hline Shofar & $\begin{array}{l}\text { Bélier } \\
\text { Chèvre sauvage } \\
\text { Kudu (Yémen) } \\
\text { Gazelle } \\
\text { Bouquetin (Syrie) }\end{array}$ \\
\hline $\begin{array}{l}\text { Cor d'appel } \\
\text { Tülök (cor d'appel hongrois) }\end{array}$ & Vache domestique \\
\hline
\end{tabular}

16 La corne seule ne permet pas forcément d'identifier quel animal (sauvage ou domestique) l'a fournie. Chamois, chèvres et bouquetins ont pu être utilisés, et les gemshorns actuels tout comme des reconstitutions de piboles ${ }^{26}$ - sont réalisés en corne de vache. 
17 Le shofar représente un cas particulier. Bien que sa symbolique soit fortement liée au bélier, la corne d'un autre animal (sauf la vache) peut être employée pourvu qu'il soit kasher; l'utilisation de kudu et de bouquetin sont des spécificités respectivement yéménite et syrienne ${ }^{27}$. Ceux vendus aux touristes en Israël proviennent de gazelles. La corne donne donc ici des informations sur la provenance de l'instrument ainsi que sur sa valeur et son rôle culturels.

Illustration 1.Pibole (ou hornpipe) avec sa capsule et son pavillon en corne de vache

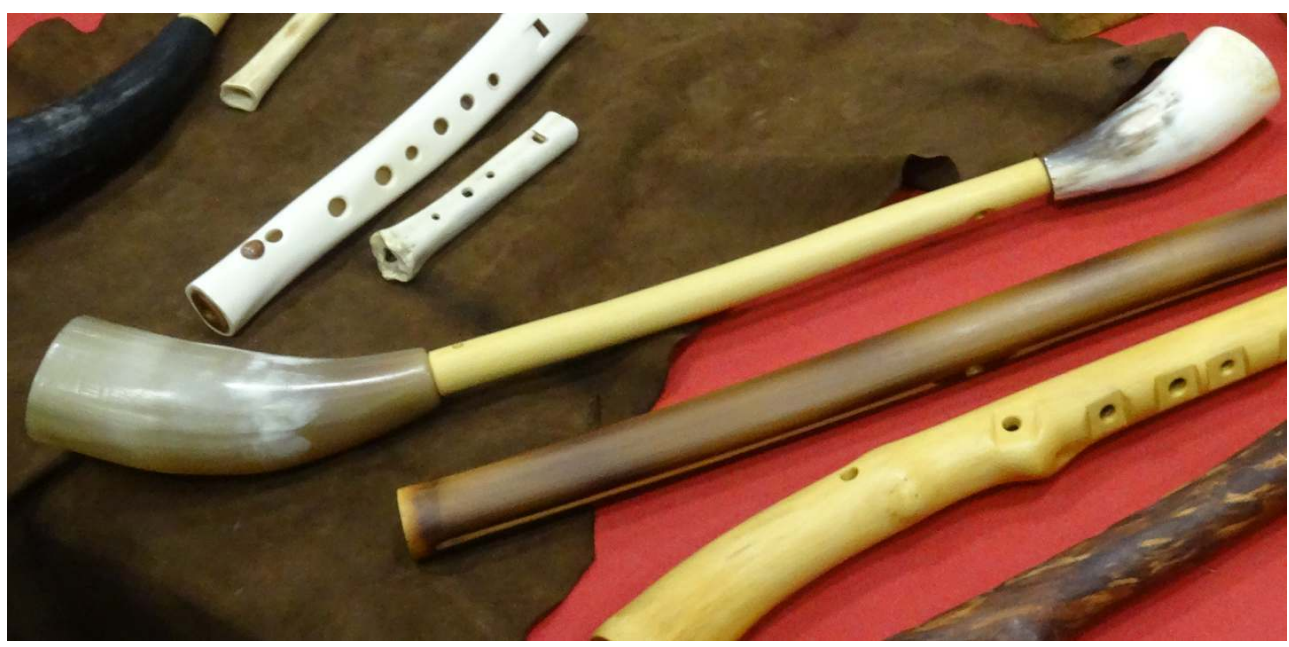

Instrument de Jeff Barbe, Salon de la musique ancienne, Vanves, 2016.

Photo Marion Weckerle 
Illustration 2. Pifana à trois trous en corne de chèvre et flûte en corne d'antilope

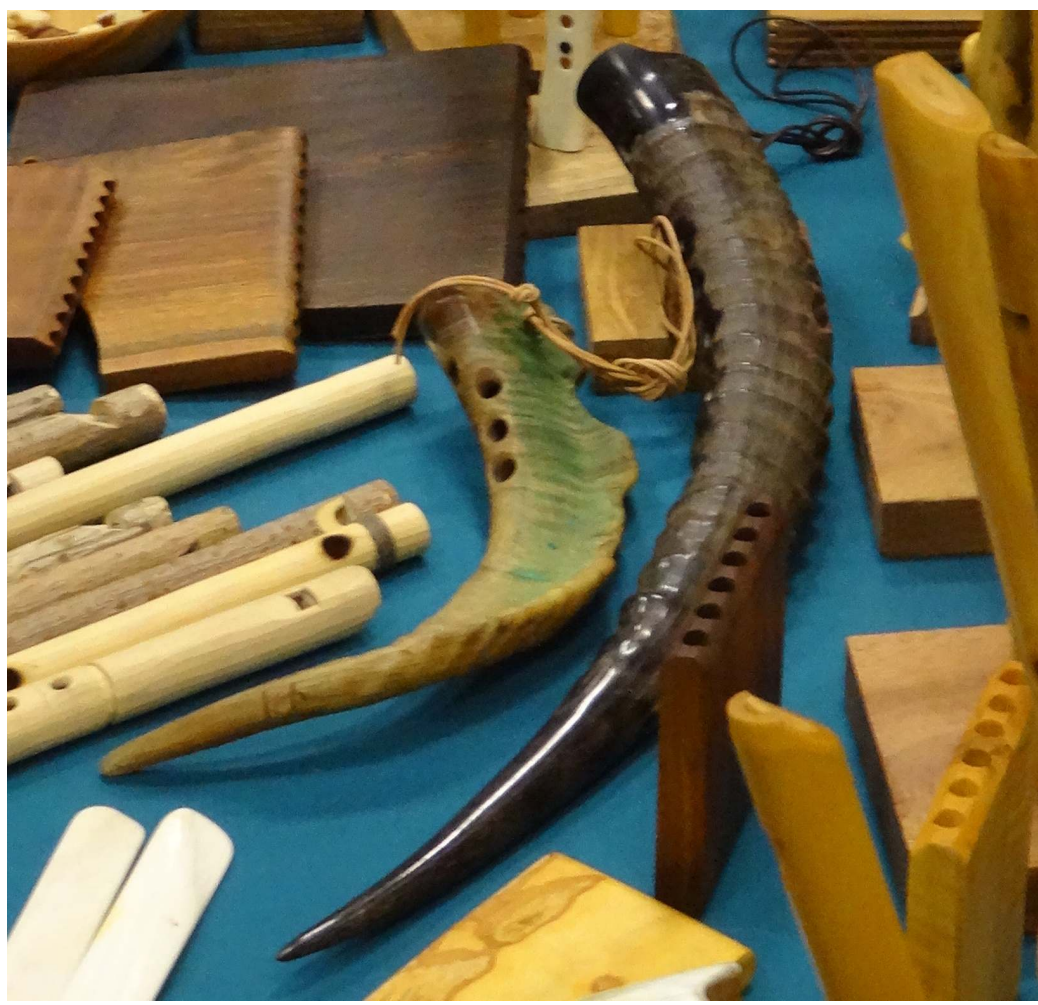

Instruments de Jean-Baptiste Talma, Salon de la musique ancienne, Vanves, 2016.

Photo Marion Weckerle

\section{Filières techniques : aérophones à biseau et aérophones à embouchure}

Les instruments en corne appartiennent à deux filières techniques différentes en terme d'émission du son. Le gemshorn, le hornpipe et la pifana sont des aérophones à biseau, percés de trous : le biseau permet la mise en place de la colonne d'air, et la longueur du tuyau (contrôlant la hauteur du son) est déterminée par le nombre de trous bouchés par les doigts.

19 Le cor d'appel et le shofar sont des aérophones à embouchure, à perce plus ou moins conique, sans trous : le son est émis par la vibration des lèvres contre l'embouchure, et limité à une ou deux notes ainsi que leurs harmoniques, limitant fortement leurs capacités mélodiques. Le cornet à bouquin du XVII ${ }^{\mathrm{e}}$ siècle, instrument à embouchure percé de trous, était un hybride de ces deux filières.

20 Ainsi, si la forme initiale de la corne détermine en partie les capacités sonores de l'instrument, le processus de transformation reste l'élément primordial, comme pour les autres vents. Pour le cor et le shofar, il est nécessaire de se demander si l'embouchure est rapportée ou non. La tradition l'interdit dans le cas du shofar, mais les choses sont plus variables pour le cor. 


\section{Vie musicale des instruments}

21 On peut isoler deux types d'utilisation des instruments, correspondant chacun à une des filières techniques (cf. tableau 2). L'utilisation de corne de vache pour les hornpipes corrobore l'idée, amenée par la littérature, qu'il s'agit d'un instrument essentiellement pastoral, que les musiciens fabriquaient eux-mêmes. On peut formuler la même hypothèse à l'encontre de la pifana et du gemshorn. Ce dernier, par contre, a peut-être connu à la Renaissance un plus grand renom, et ce jusque dans les classes sociales élevées, ayant donné son nom à un jeu d'orgue.

Quant à la filière représentée par le cor d'appel et le shofar, il s'agit d'usages essentiellement utilitaires. Les deux instruments constituent des moyens de communiquer à distance des messages en émettant des appels codés en amont, ce que font encore les bergers magyars avec le tülök. La tradition du shofar compte ainsi quatre appels, actuellement principalement utilisés lors du Nouvel An et du Yom Kippour. Plus récemment, on peut noter des compositions s'inspirant de récits bibliques ou commémorant l'Holocauste ayant intégré le shofar, pour sa force symbolique et son son, bien que de nombreux orchestres le remplacent par un bugle.

La situation est autre pour le cor d'appel: la famille des cors s'est développée pour s'adapter à divers usages musicaux, au contraire du shofar qui devait être conservé pour la pérennité du culte. L'utilisation du cor d'appel s'est donc résorbée avec la diminution de son utilité, ainsi qu'avec le développement préférentiel d'aérophones à embouchure en ivoire et en métal.

Tableau 2 : Usages répertoriés des différents instruments

\begin{tabular}{|c|c|c|c|}
\hline Instrument & Utilisation & Formation & Contexte \\
\hline Gemshorn & $\begin{array}{l}\text { Divertissement, danse } \\
\text { Divertissement }\end{array}$ & $\begin{array}{l}\text { Mêlé à d'autres } \\
\text { instruments } \\
\text { populaires } \\
\text { Consorts }\end{array}$ & $\begin{array}{l}\text { Populaire, pastoral } \\
\text { Bourgeois ou noble }\end{array}$ \\
\hline Hornpipe & Divertissement, danse & $\begin{array}{l}\text { Mêlés à d'autres } \\
\text { instruments } \\
\text { populaires }^{28}\end{array}$ & Populaire, pastoral \\
\hline Pifana & Divertissement & $\begin{array}{l}\text { Mêlé à d'autres } \\
\text { instruments } \\
\text { populaires }\end{array}$ & Populaire, pastoral \\
\hline Shofar & $\begin{array}{l}\text { Utilitaire: célébrations } \\
\text { religieuses, signalement } \\
\text { de catastrophes } \\
\text { Symbolique, } \\
\text { commémorations, } \\
\text { évocations de narrations } \\
\text { bibliques }\end{array}$ & $\begin{array}{l}\text { Isolé } \\
\text { Orchestre }\end{array}$ & $\begin{array}{l}\text { Cultes juifs: } \\
\text { Rosh Hashanah (Nouvel An), Yom } \\
\text { Kippour (Jour du Pardon) } \\
\text { Compositions orchestrales : St John } \\
\text { the Baptist, George MacFarren } \\
\text { (1873); The Apostles, Edward Elgar } \\
\text { (1903); Holocaust Requiem Kaddish } \\
\text { for Terezin, Ronald Senator (1986) }\end{array}$ \\
\hline
\end{tabular}




\begin{tabular}{|l|l|l|l|}
\hline Cor d'appel & $\begin{array}{l}\text { Utilitaire } \\
\text { Communication à } \\
\text { distance, signalement de } \\
\text { position }\end{array}$ & Isolé & $\begin{array}{l}\text { Bergers, veilleurs de nuit } \\
\text { Combats armés } \\
\text { Chasse }\end{array}$ \\
\hline
\end{tabular}

\section{Expérimentation et gestes techniques : une reconstitution de gemshorn}

Nous utilisons pour cette expérimentation le gemshorn de notre collection, réalisé en 2015 par Jean-Daniel Talma dans une corne de vache. Sa morphologie générale et sa couleur sont celles de la corne, hormis le vernis et un léger jaunissement près du bec dû à un jeu régulier de l'instrument. L'intérieur de la corne est bien sûr évidé, et, contrairement aux autres flûtes, seule l'extrémité large est ouverte. Y est inséré un bloc de bois, avec une ouverture rectangulaire dans sa partie supérieure dans lequel on souffle pour envoyer l'air sur le biseau. Le dispositif est analogue en tous points à l'embouchure d'une flûte à bec.

Illustration 3. Faces supérieure et inférieure du gemshorn avec positionnement des doigts

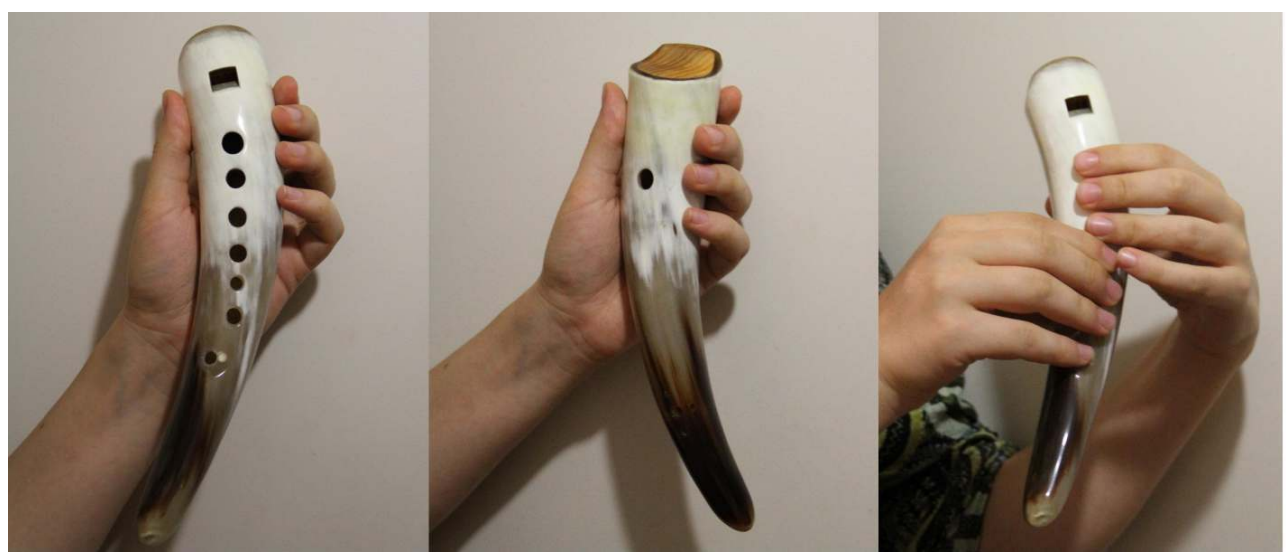

Photo Marion Weckerle

Percés sur le dessus de la corne, se trouvent sept trous de différents diamètres, dans l'axe à l'exception du dernier trou, décalé sur le côté et dans un léger creusement. Un trou pour le pouce se trouve sur la face antérieure, le tout permettant un jeu chromatique sur une neuvième. On relève d'autre part la présence d'un repentir de trou, ainsi que cinq petits trous supplémentaires au bout de l'instrument servant à l'accord en $415 \mathrm{~Hz}$ mésotonique (cf figure 1$)^{29}$.

Nous positionnons la main gauche en haut, la main droite en bas, comme sur les instruments à vent actuels. Il est également possible d'inverser les mains, mais avec un peu moins d'aisance à cause du septième trou décalé pour être atteint par l'auriculaire droit.

Tableau 3 : Fréquence en hertz, de la note jouée en appliquant les doigtés fournis dans les tablatures pour flûte à bec de Marin Mersenne, Jacob van Eyck, Sebastian Virdung, Silvestro Ganassi, et Philibert Jambe de Fer 


\begin{tabular}{|c|c|c|c|c|c|}
\hline & Mersenne & Van Eyck & Virdung & Ganassi & Jambe de Fer \\
\hline do & 415 & 415 & 415 & 415 & 415 \\
\hline do dièse & $\mathrm{x}$ & 415 & $\mathrm{x}$ & $\mathrm{x}$ & $\mathrm{x}$ \\
\hline ré & 415 & 415 & 415 & 415 & 415 \\
\hline ré dièse & $\mathrm{x}$ & 415 & $\mathrm{x}$ & $\mathrm{x}$ & $\mathrm{x}$ \\
\hline mi bémol & $\mathrm{x}$ & $\mathrm{x}$ & 415 & 415 & 415 \\
\hline $\mathrm{mi}$ & 415 & 415 & 415 & 415 & 415 \\
\hline $\mathrm{fa}$ & $\mathrm{x}$ & 415 & $\mathrm{x}$ & $\mathrm{x}$ & $\mathrm{x}$ \\
\hline fa dièse & 410 & 415 & 415 & $\mathrm{x}$ & 410 \\
\hline sol & 410 & 415 & 415 & 415 & 415 \\
\hline sol dièse & $\mathrm{x}$ & 415 & 415 & 410 & 410 \\
\hline la & 410 & 415 & 415 & 415 & 415 \\
\hline la dièse & $\mathrm{x}$ & 415 & $\mathrm{x}$ & $\mathrm{x}$ & $\mathrm{x}$ \\
\hline si bémol & 410 & 415 & 415 & 415 & 410 \\
\hline si & 410 & 415 & 415 & 415 & 415 \\
\hline do (2e octave) & $\mathrm{x}$ & 415 & 415 & 415 & 410 \\
\hline do dièse (2e octave) & $\mathrm{x}$ & 415 & 415 & $\mathrm{x}$ & $\mathrm{x}$ \\
\hline ré bémol (2e octave) & $\mathrm{x}$ & $\mathrm{x}$ & $\mathrm{x}$ & $\mathrm{x}$ & $\mathrm{x}$ \\
\hline ré (2e octave) & $\mathrm{x}$ & 415 & 430 & 415 & 415 \\
\hline
\end{tabular}

Plusieurs systèmes de doigtés peuvent coexister. Afin de monter une note d'un demi-ton, il est possible de déboucher à moitié le dernier trou bouché : cette technique est la plus simple mais aussi la moins juste. La technique des doigtés dits " de fourche » permet d'obtenir des intervalles plus justes, en avançant le dernier doigt d'un trou. Toutefois, si les doigtés sont généralement identiques à ceux décrits dans les traités de flûte à bec, les tablatures ne sont pas rigoureusement interchangeables : changer de matériel implique d'adapter ses gestes. La plupart des doigtés disponibles fonctionnent sur un instrument accordé en $415 \mathrm{~Hz}$, c'est-à-dire l'un des diapasons "standard » baroques, tels que notre gemshorn, mais certaines notes ne sont pas fournies ou les doigtés ne sont pas applicables au gemshorn, ce que nous représentons par un « $\mathrm{x}$ ». La tablature de Van Eyck donne le plus de possibilités, tandis que celle de Mersenne convient clairement mieux à un instrument accordé en $410 \mathrm{~Hz}$. Ceci étant, le répertoire pour flûte à bec des $\mathrm{XVI}^{\mathrm{e}}$ et du 
$\mathrm{XVII}^{\mathrm{e}}$ siècles peut tout à fait être interprété au gemshorn, avec quelques adaptations minimes ${ }^{30}$. Nous n'avons malheureusement pas pu expérimenter sur d'autres tailles de gemshörner ou sur les autres instruments décrits dans l'article, les rares facteurs ne les prêtant que dans la perspective d'un achat.

\section{Étude d'instruments conservés en musées} dans une grande partie de l'Europe, et pas forcément dans leur pays d'origine. En particulier, plusieurs shofars viennent de régions où la religion juive a été historiquement très présente. Il est d'autre part difficile de tracer les instruments, anonymes et dont le contexte d'acquisition est inconnu. La plupart oscillent entre le XVIII et le début du XX siècle. Nous ne disposons donc d'aucun gemshorn historique (hors celui du Musikinstrumenten Museum de Berlin, très mal documenté) et aucune pifana. Les trois pigborns survivants connus ${ }^{31}$ se trouvent au National Museum of Wales et ont été étudiés par ailleurs ${ }^{32}$.

31 À travers un corpus de vingt-et-un shofars, on observe une certaine diversité, alors même que sa symbolique est censée le rendre immuable. Dans treize cas, la corne rectiligne s'incurve vers le haut presque à angle droit à son extrémité pour former le pavillon. Dans l'autre cas, les torsades naturelles de la corne ont été conservées, ce qui nous indique deux écoles esthétiques pour l'apprêt initial de la corne, sans incidence sur le son. La corne peut être redressée puis lissée par chauffage puis par grattage ou raclage, avant de former l'embouchure et une perce conique dans la cavité naturelle ${ }^{33}$. Les bords du pavillon peuvent soit être coupés simplement soit présenter un motif décoratif ${ }^{34}$, celui-ci pouvant même s'étendre à tout l'instrument ${ }^{35}$. On peut également constater que les shofars, comme prescrit par la tradition hébraïque, sont tous constitués uniquement de la corne, sans élément rapporté comme une embouchure amovible. L'embouchure est formée par la pointe de la corne sciée, dont les bords sont éventuellement sculptés en une forme circulaire. variées, sans justification : olifant, corne (le plus répandu et moins précis), corne d'appel, et cor moyen, long, français, de veilleur de nuit, de berger, ou de protection. On constate toutefois l'existence de noms de métiers ou de fonctions. Un instrument répertorié sous le nom de «corne » au Musée de la Castre ${ }^{36}$ est probablement un shofar «droit » tel que décrit plus haut. La corne d'appe ${ }^{37}$ se distingue en étant à la fois le plus ancien répertorié (la date 1420 y est sculptée) et le plus richement orné avec des scènes macabres et de chasse ; il pourrait s'agir d'un instrument avant tout décoratif, ou destiné à une personne fortunée.

Contrairement aux shofarot, les cors ne sont pas exempts de décorations mais aussi de modifications utilitaires, impliquant des niveaux de sophistications très variables, les plus simples étant généralement désignés comme cors de berger. Trois des cornes, non datées, ont une embouchure externe (deux en métal, probablement argent, et une en ivoire) ; il s'agit probablement d'instruments à vocation utilitaire, ce qui expliquerait l'embouchure externe pour faciliter le jeu. De plus, trois exemplaires ont un pavillon en métal ajouté sur la corne naturelle. Il paraît peu probable que de tels pavillons eussent été ajoutés pour

e-Phaïstos, V-1 2016 | 2018 
amplifier le son, compte tenu de leur petite taille, mais plutôt pour « cuivrer » son timbre, peut-être pour répondre à une préférence esthétique de leur époque de fabrication.

\section{Conclusion}

Il ressort de l'étude des filières techniques de ces aérophones en corne une grande diversité de construction, de gestes techniques et d'usages issus pourtant de mêmes logiques techniques. Ces formes, méconnues ou considérées comme peu élaborées, sont ainsi à l'origine de lignées d'instruments récents plus familiers.

La reconstitution et l'expérimentation donnent des éléments d'identification pour les instruments, et des précisions quant à leur mode de jeu et tout particulièrement leur timbre. La pratique historiquement informée des instruments anciens devrait même à notre sens passer systématiquement par ces méthodes, et leur application aux pifanas et hornpipe permettrait de combler des lacunes, comme les problèmes d'identification des instruments incomplets mis au jour par l'archéologie, la méconnaissance des techniques de fabrication historiques de ces instruments, ainsi que de leur répertoire.

Le développement de bases de données sur le web 2.0 permet de combler en partie les difficultés d'accès aux instruments, notamment pour le shofar et le cor d'appel. Cependant, le référentiel utilisé a été pensé comme un catalogue indicatif et non comme un réel outil de recherche, puisque des informations basiques sur les instruments manquent régulièrement et que les clichés sont peu nombreux.

Nous avons toutefois pu enrichir la connaissance du complexe technique des shofarot puisque la matière utilisée et les transformations de la corne donnent des informations sur la provenance et le rôle socio-culturel de celle-ci. Les cors d'appel eux, montrent une grande variété de transformations techniques superficielles basées sur des critères de commodité et d'esthétique visuelle et peut-être sonore. Les cors peuvent ainsi se retrouver dans de très nombreux groupes sociaux au contraire des shofarot à usage principalement symbolique et religieux, et aux instruments à biseau liés à la musique populaire et pastorale.

\section{NOTES}

1. Il existe deux types de cornes dans le monde animal : la corne entièrement formée de kératine telle celle du rhinocéros, et celle formée d'une couche de kératine recouvrant une cheville osseuse, qui est la plus répandue et celle traitée ici.

2. SADIE Stanley, The New Grove Dictionary of Musical Instruments, Londres, MacMillan, 1984, 3 vol.

3. «Cornicyll» en gaélique.

4. IORWERTH Peate, «Welsh Musical Instruments", Man, vol. 47, 1947, p. 21-25; LANGWILL Lindesay, "The Stock-and- Horn", Proceedings of the Society of Antiquaries of Scotland, vol.84, novembre 1950, p. 173-180. 
5. L'ajout d'un sac recevant une réserve d'air pour permettre l'émission d'un bourdon ou de son dans plusieurs tuyaux à la fois est, lui, une caractéristique des bagpipes, c'est-à-dire de la catégorie des cornemuses.

6. SACHS Curt, «Das Gemshorn », Zeitschrift für Musikwissenschaft, vol. 1 n³, décembre 1918, p. 153-156.

7. FÉTIS François-Joseph, Histoire générale de la musique, Paris, Firmin-Didot, 1869, 5 vol.

8. FITZPATRICK Horace, "The Gemshorn: a reconstruction", Proceedings of the Royal Musical Association, vol. 99, 1972-1973, p. 1-14.

9. PARKINSON Andrew, «Guesswork and the Gemshorn », Early Music, vol. 9 n¹, janvier 1981, p. 43-46.

10. BALFOUR Henry, "The Old British «Pibcorn » or " Hornpipe » and its Affinities ", Journal of the Anthropological Institute of Great Britain and Ireland, vol. 20, 1890, p. 142-154; TAMBOER Annemies, «Excavated Sounds. Reconstruction of Lyre, Hornpipe and Other Archaeological Instruments for Education and Research », Orient-Archäologie Band,10, 2002, p. 237-243.

11. Le concept de lignée technique, tel que développé au Centre d'histoire des techniques de l'Université Paris 1, se définit comme une parenté entre les objets ou leur fonction quel que soit leur fonctionnement technique. Bien que ce terme ne soit pas employé dans le texte, il s'applique donc bien aux « arbres généalogiques » groupant des instruments de musique " parents ».

12. LACHEZE Cyril, La tenue du violon à l'époque baroque, mémoire de Master 2 d'Histoire des techniques (dir. Anne-Françoise Garçon), Paris, Université Paris 1, 2013.

13. LE VRAUX Denis, «La muse en os du château de Mayenne ", Rencontres de musique médiévale, Largentière, 2011, https://www.youtube.com/watch?v=edFw_hK-Qtk, dernière consultation le 15 décembre 2016 ; LE VRAUX Denis, «La pibole de Pouzauges », mis en ligne le 15 septembre 2016, https://www.youtube.com/watch?v=TMfQzfZVjJM, dernière consultation le 15 décembre 2016.

14. LEJEUNE Jérôme (éd.), Guide des instruments anciens, s.l., Ricercar, 2009.

15. Schmuhl Hans Von Boje E., LUSTIG Monika (éds.), Jagd-und Waldhörner: Geschichte und musikalische Nutzung, 25 Musikinstrumentenbau-Symposium : Michaelstein, 8. bis 10. Oktober 2004, Augsburg, Wissner-Verlag, 2006 ; LAURENTY Jean-Sébastien , «La systématique des aérophones en Afrique centrale ", Koninklijk Museum voor Midden-Afrika, n7, 1974; Klaus Sabine Katharina, Trumpets and other High Brass, Volume 1: A History Inspired by the Joe R. and Joella F. Utley Collection: Instruments of the Single Harmonic Series, Vermillion, SD: National Music Museum, 2012.

16. MILLER Malcolm, "The Shofar and its Symbolism », Historic Brass Society Journal, vol. 14, 2002, p. 83-113 ; LEMAIRE Frans, Le destin juif et la musique, Paris, Fayard, 2001 ; MONTAGU Jeremy, Musical instruments of the Bible, London, Scarecrow Press, 2002.

17. BRAUN Joachim, Music in Ancien Israel/Palestine, archeological, written, and comparative sources, Grand Rapids, Eerdmans, 2002; LUSCINIUS Ottmar, Musurgia seupraxis musicœe, Strasbourg, Ioannem Schottum, 1536, p. 20; VIRDUNG Sebastian, Musica getutscht, Bâle, s.n., 1511, s.p.

18. MONTAGU Jeremy, Horns and Trumpets of the World: an Illustrated Guide, Lanhma, Rowman \& Littlefield Publishers, 2014.

19. AGRICOLA Martin, Musica instrumentalis Deudsch, Wittenberg, Georg Rhaw, 1529, s.p.; BALFOUR Henry, «The Old British «Pibcorn » or « Hornpipe » and its Affinities », Journal of the Anthropological Institute of Great Britain and Ireland, vol. 20, 1890, p. 142-154.

20. «Cornat » en ancien français traduit par cor, et « olifan » pour olifant.

21. Troisième volet de sa tétralogie L'Anneau des Nibelungen, dans lequel Siegfried poussé par la solitude, sonne du cor dans la forêt et entre ainsi en contact avec un oiseau.

22. Premier volet de la trilogie littéraire Le Seigneur des Anneaux, dans lequel une analogie flagrante avec la mort de Roland peut être établie, puisque Boromir sonne de son cor en corne de vache pour appeler des renforts alors qu'il lutte seul contre une armée d'Orcs et meurt héroïquement, son cor fendu en deux.

23. Anonyme, The Compaynt of Scotland, $1^{\text {ère }}$ éd. 1548, Edinburgh, John Leyden, 1801, p. 101. 
24. Dont celles du Musée de la Musique de Paris, du Gemanisches Nationamuseum de Nuremberg ou encore du Musik \& Teatermuseet de Stockholm, http://www.mimo-international.com/, dernière consultation le 2 décembre 2016.

25. Ensemble des moyens nécessaires (matière première, énergie, infrastructures, savoir-faire et transport) à la réalisation.

26. Par exemple celle de Jeff Barbe que nous avons pu voir aux Journées de la musique ancienne de Vanves de 2016.

27. MONTAGU Jeremy, Horns and trumpets..., op.cit., p 55.

28. Cornemuse (bagpipe), gemshorn, flûte à bec, vièle, trompette dans The Complaynt of Scotland.

29. Comme il est physiquement impossible que tous les intervalles, en jouant les notes deux à deux, soient justes, on choisit des compromis : ce qu'on appelle des tempéraments.

30. VAN EYCK Jacob , Der Fluyten Lusthof, Amsterdam, 1649; PRAETORIUS Michael, Syntagma Musicum, Wittenberg, Johannes Richter, 1615.

31. Sous-genre de hornpipe.

32. LANGWILL Lyndesay, "The Stock-and- Horn ", Proceedings of the Society of Antiquaries of Scotland, vol.lxxxiv, 1950, p. 173-180.

33. MONTAGU Jeremy, Horns and trumpets..., op.cit.

34. Exemplaire F 725 du Scenkonst Museet de Stockholm.

35. Exemplaire F 724 du Scenkonst Museet de Stockholm.

36. Numéro 2009.0.831, Cannes.

37. E. 294, Musée de la Musique de Paris.

\section{RÉSUMÉS}

Les instruments de musique à vent médiévaux et modernes sont le plus souvent étudiés en tant qu'ancêtres d'instruments actuels. Ils sont peu envisagés sous l'angle des filières techniques et leurs complexes techniques respectifs sont donc relativement peu décrits. Or, ceux-ci sont susceptibles de fournir des indications pour la pratique de la musique historiquement informée, mais aussi pour mieux comprendre les instruments anciens en tant qu'objets historiques et archéologiques. Dans cet article, nous examinons cinq instruments à vent européens et procheorientaux appartenant à la filière technique des instruments en corne, tout en répondant à des techniques de jeu musicales et à des usages sociaux propres. Il ressort de l'analyse que, bien que la corne employée détermine en partie les capacités de l'instrument, c'est le processus de transformation de la corne qui est déterminant. On peut donc obtenir avec ce même matériau deux filières techniques différentes en terme d'émission du son: les aérophones à biseau (gemshorn, pifana, hornpipe) et les aérophones à embouchure (cor d'appel et shofar). Les instruments de ces deux filières correspondent également à deux grands types d'utilisation : la première axée sur le divertissement et la danse, le second sur des fonctions utilitaires et symboliques. Enfin, le choix et la transformation de la corne elle-même donne des indications sur le complexe technique lié à l'instrument ainsi que sur son emploi. Dans le cas du gemshorn, dont le répertoire historique est inconnu, l'apprêt de la corne le rapproche de la flûte à bec. Nous avons constaté par l'expérimentation que son répertoire pouvait être joué, moyennant des adaptations. Les registres ne coïncident pas exactement, et les doigtés utilisables au gemshorn sont proches des systèmes de doigtés des flûtes à bec des $\mathrm{XVI}^{\mathrm{e}}$ et $\mathrm{XVII}^{\mathrm{e}}$ siècles mais non 
interchangeables. Changer de matériau de base implique donc de s'adapter et pas d'imiter. Dans le cas du shofar et du cor d'appel, la transformation appliquée aux cornes répond à des critères esthétiques et à des règles cultuelles : elle constitue des indicateurs sur l'appartenance sociale des instruments examinés.

Medieval and modern wind musical instruments are studied, most of the time, as ancestors from current instruments. They are little studied under the angle of the technical sectors and thus their complex techniques are relatively little described. Yet, these may provide indications for the practice of the historically informed music, but also for a better understanding of the ancient musical instruments as historic and archaeological objects. In this article, we examine five European and Near Eastern wind instruments belonging to the technical sector of instruments in horn, with their specific playing techniques and social uses: the gemshorn, the pifana, the shofar, the hornpipe and the call horn. The analysis shows that, although the type of horn partly determines the capacities of the instrument, it is transformation process of the horn which is determining. Thus, we can obtain,with the same material, two distinct technical sectors in terms of sound production: bevel aerophones (gemshorn, pifana, hornpipe) and mouthpiece aerophones (call horn and shofar). The instruments of these two sectors also belong to two distinct types of use: the first focused on entertainment and dance, the second on utilitarian and symbolic functions. In addition, the choice and the transformation of the horn itself gives indications on the technical complex related to the instrument, but also on its use. In the gemshorn's case, of which the historical repertoire in unknown, the horn preparation brings it close to the recorder, and we noted by experimenting that the recorder Renaissance and early Baroque repertoire can be played on the gemshorn with a few adjustments. The registers do not exactly match, and the fingerings of the XVI ${ }^{\text {th }}$ and XVII ${ }^{\text {th }}$ recorders are close to the fingerings appliable on the gemshorn although not exchangeable. To change the basic material thus implies an adaptation of the playing techniques and not an imitation. In the case of the shofar and the call horn, the transformation applied to the horns answers esthetic criteria and worship rules: they are indicators of the social place of the examined instruments.

\section{INDEX}

Thèmes : Un objet une technique

Mots-clés : Histoire des techniques, filière technique, lignée technique, instrument de musique, organologie, gemshorn, pifana, shofar, olifant, pibole, hornpipe, corne

Keywords : History of technology, technical sector, technical lieange, musical instrument, organology, gemshorn, pifana, shofar, olifant, hornpipe, animal horn

\section{AUTEUR}

\section{MARION WECKERLE}

Marion Weckerle est doctorante en histoire des techniques sous la direction du professeur AnneFrançoise Garçon, au Centre d'Histoire des techniques de l'Université Paris 1 Panthéon-Sorbonne (IHMC UMR 8066). Ses recherches portent sur l'innovation et les transferts techniques dans une approche systémique, qu'elle analyse à travers le cas des hydravions au début du XXe siècle. Elle est également formée à la musique ancienne, à laquelle elle applique les mêmes thématiques. 